\title{
Perfil dos pesquisadores bolsistas de produtividade científica em Psicologia do Conselho Nacional de Desenvolvimento Científico e Tecnológico
}

\section{The profile of scientific productivity of Brazilian Psychology research scholars of the Conselho Nacional de Desenvolvimento Científico e Tecnológico}

\author{
João Luís Almeida WEBER ${ }^{1}$ \\ Carine Capra RAMOS ${ }^{1}$ \\ Ariela MESTER ${ }^{1}$ \\ Daniele LINDERN ${ }^{1}$ \\ Kyndze Rodrigues HÖRLLE \\ Caroline dos Santos de SOUZA ${ }^{1}$ \\ Adolfo PIZZINATO ${ }^{1,2}$ \\ Kátia Bones ROCHA ${ }^{2}$
}

\begin{abstract}
Resumo
O objetivo desta pesquisa foi traçar o perfil dos pesquisadores bolsistas de Produtividade em Psicologia do Conselho Nacional de Desenvolvimento Científico e Tecnológico no triênio 2009-2011. O método utilizado foi de caráter transversal, descritivo, correlacional e de associação. Por meio de um levantamento de dados presentes nos currículos Lattes dos pesquisadores listados no site do Conselho Nacional de Desenvolvimento Científico e Tecnológico, buscou-se identificar relação entre a categoria de bolsa, a quantidade e a qualidade de suas produções. Os achados revelam diversidade, não permitindo traçar um perfil único de bolsistas. Predominam os bolsistas de categoria 2, distribuídos, em sua maioria, nas áreas de interesse da Psicologia do Desenvolvimento e Social. Verificou-se que, apesar de a qualidade das publicações acompanhar a categoria da bolsa, ainda predomina a quantidade em relação à qualidade das publicações, fenômeno conhecido como "produtivismo".
\end{abstract}

Palavras-chave: Bolsista produtividade; Pesquisa em Psicologia; Psicologia no Brasil.

\begin{abstract}
The aim of this study was to draw the profile of the psychology researchers funded with scientific productivity grants from the Conselho Nacional de Desenvolvimento Científico e Tecnológico from 2009-2011. We used a cross-sectional, descriptive and correlational method. Through a data survey using the Curriculum Lattes platform of all the researchers listed on the website of Conselho Nacional de Desenvolvimento Científico e Tecnológico, the purpose was to identify

$\nabla \nabla \nabla$

1 Pontifícia Universidade Católica do Rio Grande do Sul, Faculdade de Psicologia, Curso de Psicologia. Porto Alegre, RS, Brasil.

2 Pontifícia Universidade Católica do Rio Grande do Sul, Faculdade de Psicologia, Programa de Pós-Graduação em Psicologia. Av. Ipiranga, 6681, Prédio 11, Sala 918, 90619-900, Porto Alegre, RS, Brasil. Correspondência para/Correspondence to: KB ROCHA. E-mail: <katia.rocha@pucrs.br>.
\end{abstract}


the relation between type of grant, quantity and quality of their production. The findings reveal diversity, which did not enable us to establish a unique profile of the researchers. Most researchers have a type-2 research grant and their interest is mainly distributed in Developmental and Social Psychology. Although the quality of publications is related to the type of grant, there is still a predominance of quantity over quality of publications, a phenomenon called "productivism".

Keywords: Scientific productivity grants; Research in Psychology; Psychology in Brazil.

No Brasil, atualmente, os principais órgãos públicos de fomento à pesquisa científica de caráter acadêmico são o Conselho Nacional de Desenvolvimento Científico e Tecnológico (CNPq) e a Coordenação de Aperfeiçoamento de Pessoal de Nível Superior (Capes). O primeiro é responsável pela formação e qualificação de pesquisadores, enquanto o segundo avalia, divulga, promove, fomenta e fornece recursos humanos e financeiros, objetivando contribuir para a ciência (Conselho Nacional de Desenvolvimento Científico e Tecnológico [CNPq], 2006a; Coordenação de Aperfeiçoamento de Pessoal de Nível Superior [Capes], 2006).

Nos últimos anos, o maior investimento público em pesquisas no Brasil se deu nas áreas de Ciências Biológicas (18,23\%), Agrárias (15,72\%), Exatas e da Terra (14,03\%), Engenharias (13,23\%), Saúde (12,64\%) e Humanas (12,58\%). Dentre as Ciências Humanas, a Psicologia recebe o segundo maior investimento $(14,21 \%)$, correspondente a $1,79 \%$ do total nacional, ficando abaixo somente da Educação (35,43\%). Esse investimento se expressa no número de projetos beneficiados nas duas áreas, no total de 221 na Psicologia e 551 na Educação (CNPq, 2006b).

Dentre os benefícios principais e de maior relevância acadêmica, destaca-se a concessão de bolsas de produtividade em pesquisa do CNPq. Reservada a doutores que se destaquem em suas atividades de produção científica, a bolsa possibilita a dedicação à pesquisa com maiores recursos e garantias de reconhecimento. Tais bolsas de produtividade devem ser solicitadas pelo próprio pesquisador por meio de um formulário de proposta on-line, de acordo com calendário anualmente divulgado pelo CNPq. Os critérios avaliados pelos Comitês de Assessoramento do CNPq para solicitação da bolsa são: formação de recursos humanos,

2 em nível de Pós-Graduação; participação principal ou coordenação de projetos de pesquisa; produção científica; participação em atividades de gestão científica e editoriais; administração de núcleos de excelência científica, tecnológica e de instituições; contribuição científica e tecnológica e para inovação (CNPq, 2006c).

As bolsas são divididas em três categorias: Pesquisador 1, Pesquisador 2 e Pesquisador Sênior. Um dos pré-requisitos para se enquadrar na primeira categoria é ter concluído o doutorado há pelo menos 8 anos, enquanto para a segunda categoria deve-se tê-lo concluído há no mínimo 3 anos. Já o Pesquisador Sênior necessita de, no mínimo, 15 anos com bolsa na categoria 1 (A ou B) (CNPq, 2006c).

Na categoria 1 dá-se uma divisão por níveis (A, B, C e D), com base na comparação com outros pesquisadores e nos dados dos últimos dez anos. Além disso, são avaliadas a produção científica, a contribuição ou participação em projetos de pesquisa, e a inserção nacional e internacional. Para se enquadrar no nível A, por exemplo, o pesquisador deve alcançar contínua excelência na produção, demonstrando grande destaque em sua área de atuação, além de liderar um grupo de pesquisa consolidado. Já na categoria 2, a produção avaliada para concessão da bolsa é referente aos últimos 5 anos, e não há diferenciação de níveis (na categoria 2, foi implementada a bolsa 2F, que tem cunho transitório e objetiva fortalecer os pesquisadores de cursos emergentes) (CNPq, 2006c).

A área das Ciências Humanas totaliza 1654 pesquisadores bolsistas, sendo a Psicologia a segunda subárea com maior número (295), novamente atrás apenas da Educação (390). Esse número pode ter sido influenciado pelo aumento dos Programas de Pós-Graduação (PPG) em Psicologia, passando de 13 para 61 entre 1976 e 2007 (Lo Bianco, Almeida, Koller, \& Paiva, 2010). Acompanhando esse 
aumento dos PPG em Psicologia, a produção bibliográfica também tem se mostrado crescente na área nos últimos anos (Costa, Amorim, Pessanha, \& Yamamoto, 2012).

O novo plano nacional de pós-graduação, que compreende o período de 2011-2020, tem como uma de suas principais metas aumentar ainda mais o número de doutores no Brasil. Em relação a 2010, o Brasil busca dobrar o número de doutores até 2020; para tanto, o número de bolsas concedidas pela Capes e pelo CNPq tem previsão de crescimento de $256 \%$. O aumento, desejável, da formação de doutores seria sustentado pela maior oferta de bolsas, tendo em vista que atualmente cerca de metade dos alunos da pós-graduação não as recebem (Capes, 2010). Assim, mais pesquisadores seriam atraídos a ingressar na pós-graduação, a garantir sua titulação e, consequentemente, a disputar o fomento para a pesquisa, especialmente a Bolsa de Produtividade.

A partir do "III Plano Nacional de Pós-Graduação (1986-1989)", passou-se a estimular mais a autonomia em nível nacional, tanto na tecnologia quanto na ciência, dos PPG. O foco na docência, que era central, foi transferido para a pesquisa, que remete diretamente à produção e à divulgação do conhecimento. Essa mudança de foco e a importância delegada para a produção científica influenciam a avaliação global dos PPG de maneira cada vez mais forte (Yamamoto, Tourinho, Bastos, \& Menandro, 2012).

O reconhecimento do peso talvez desproporcional da produção científica levou a Capes, no triênio 2004-2006, a alterar as ponderações dos diversos elementos da avaliação, indicando a equiparação dos quesitos referentes à formação (corpo discente, teses e dissertações) e à produção científica que, combinados, deveriam responder por $70 \%$ da avaliação final. Na área da Psicologia, ambos os quesitos tiveram o mesmo peso, respondendo, cada um deles, por 35\% da nota final, na Avaliação Trienal de 2010 (Yamamoto et al., 2012).

A descrição feita anteriormente pode comprovar que o aumento do número de PPG, de alunos, e o crescimento do foco em relação à produção científica - assim como a revisão de seus quesitos de avaliação -, posicionam a Psicologia como uma área com qualidade na produção de conhecimento.

Buscando entender a relação entre quantidade e qualidade dessa produção, o objetivo desta pesquisa foi traçar o perfil dos pesquisadores bolsistas de produtividade em Psicologia do CNPq e sua produção acadêmica durante o triênio 2009-2011.

\section{Método}

O presente estudo tem um caráter transversal, descritivo e correlacional.

\section{Participantes}

Os participantes do presente estudo são os currículos Lattes dos 295 pesquisadores bolsistas de produtividade científica em Psicologia do CNPq no ano de 2011.

\section{Instrumentos}

A partir da análise dos currículos Lattes, foram selecionadas as seguintes informações: categoria da bolsa de produtividade (Sênior, 1A, 1B, 1C, 1D, 2 e 2F); estado e Instituição de Ensino Superior (IES) do pesquisador; tempo de conclusão de doutorado; áreas de interesse (número total de áreas e área principal); número de publicações (artigos - Qualis A1, A2, B1, B2, B3, B4, B5, C e sem Qualis; livros e capítulos de livros); número de pós-doutorandos, doutorandos, mestrandos e bolsistas de iniciação científica que orienta; nome dos periódicos em que publicou.

Por último, foi analisado o índice de Hirsch (h) desses bolsistas, o qual quantifica numericamente o impacto acadêmico de um pesquisador, combinando quantidade com qualidade das publicações (Harzing, 2008). A escolha desse indicador, dentre outras possibilidades, como a presença no ISI Web of Knowledge (Thomaz, Assad, \& Moreira, 2011), deve-se à sua facilidade de acesso e ao mapeamento mais abrangente de bases de dados (especialmente as de livre acesso) acadêmicas, pois utiliza estratégias de busca e mapeamento da Internet, para além das bases de dados assinadas. 


\section{Procedimentos}

Os dados foram tabulados no programa Statistical Package for the Social Sciences (SPSS) versão 17.0 para Windows. Para realizar a análise de associação entre o tipo de bolsa do pesquisador e as publicações nos diferentes Qualis, foram calculados modelos de regressão logística bivariados, mediante o cálculo de Odds Ratio (OR) e Intervalos de Confiança 95\% (IC 95\%). Para tanto, foi utilizado o programa Stata versão 9. Para a análise do índice de Hirsch utilizou-se o software Publish or Perish versão 3.7 para Windows. Os dados analisados foram coletados em maio de 2012.

\section{Resultados e Discussão}

A partir do levantamento de dados dos currículos Lattes dos pesquisadores de Psicologia, constatou-se que o maior número de bolsas de produtividade é destinado à categoria 2 (58,3\%), seguida pela categoria 1D (13,2\%). Em último lugar aparece a categoria sênior, com somente uma representante $(0,3 \%)$, o que implica, evidentemente, em relativização do uso dessa categoria na análise. Essa proporção também é observada nos bolsistas de produtividade do CNPq de outras áreas, como Medicina, Odontologia e Saúde Coletiva (Mendes, Martelli, Souza, Quirino Filho, \& Martelli Júnior, 2010).

Analisando a Tabela 1, em que consta o tempo médio de obtenção do título de doutor, é possível destacar que as faixas de 11-15 e 21-30 anos concentram juntas mais da metade dos pesquisadores (55,9\%). Dos 295 pesquisadores, 172 estão concentrados na categoria 2, e 116 na categoria 1 (1A, 1B, 1 C e 1D). Contudo, 31 bolsistas da categoria 2 completaram o doutorado há 21 anos ou mais, considerando-se que já poderiam estar enquadrados em uma categoria superior (pois para se enquadrar na categoria 1 o pesquisador precisa ter concluído o doutorado há pelo menos 8 anos), o que denota que não é apenas o tempo de doutoramento o fator-chave para a progressão no sistema. Verificou-se, inclusive, que a média de publicações de artigos científicos de tais bolsistas - que completaram doutorado há pelo menos 21 anos e permanecem na categoria 2 -, em periódicos, foi muito inferior à média geral da categoria (9,2 publicações), durante o triênio 2009-2011 (5,26 publicações). Apenas 3,0\% dos bolsistas concluíram o doutorado há cinco anos ou menos, estando estes presentes somente na categoria 2 - o que é esperado, já que somente a categoria 2 pode abranger doutores com até 5 anos de doutorado. Observa-se também que

Tabela 1

Distribuição dos pesquisadores bolsistas de produtividade em Psicologia segundo tempo de conclusão do doutorado e sexo na categorização do CNPq no triênio 2009-2011

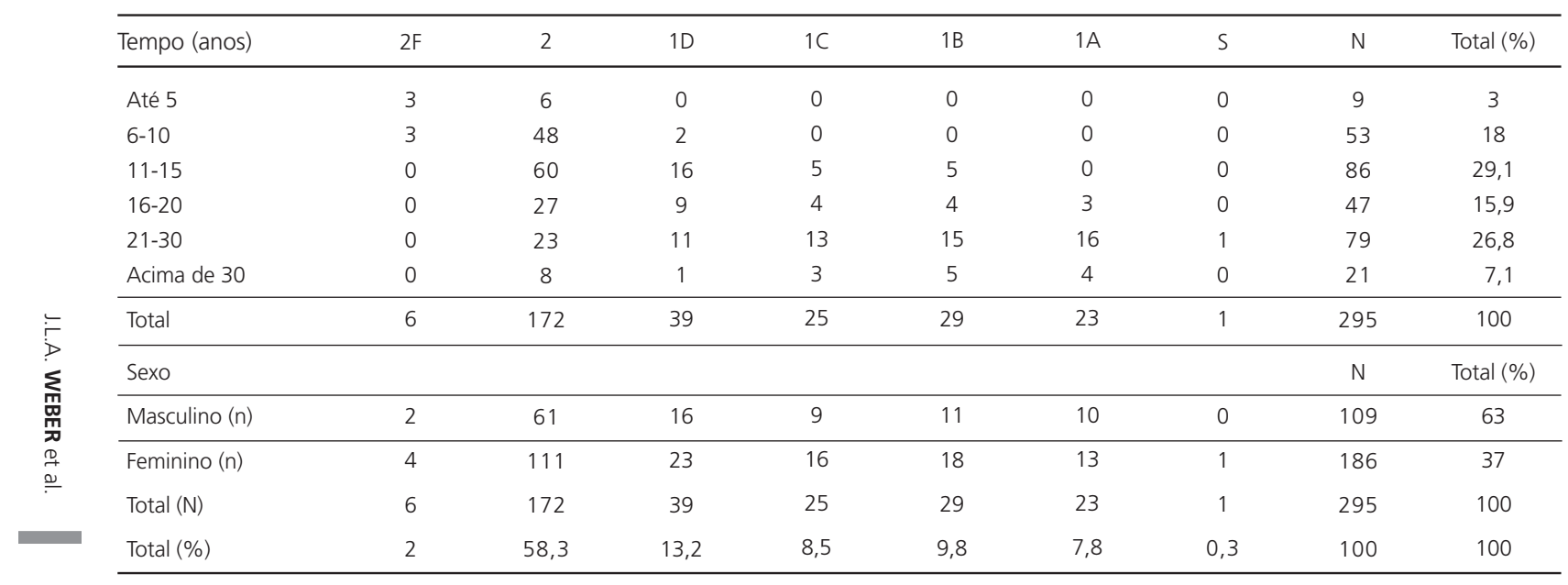

4 Nota: CNPq: Conselho Nacional de Desenvolvimento Científico e Tecnológico. 
as categorias 1C, 1B, 1A e Sênior (S) apresentam apenas bolsistas com pelo menos 11 anos de doutorado, sendo as duas últimas com, no mínimo, 16 anos.

Além disso, dos 295 bolsistas de produtividade analisados, 109 são do sexo masculino $(37,0 \%)$ e 186 do sexo feminino $(63,0 \%)$ (Tabela 1). Contudo, apesar do predomínio do sexo feminino em todas as sete categorias de bolsas, essa diferença é muito inferior à distribuição de profissionais psicólogos por sexo, uma vez que o total de psicólogas registradas nos Conselhos Regionais no Brasil é de 191 222, representando 88,3\% dos 216419 psicólogos brasileiros (Conselho Federal de Psicologia, 2012). Esse dado demonstra que, apesar do crescimento e maior inserção das mulheres na comunidade científica nacional, o predomínio ainda é do sexo masculino (Leta, 2003).

A análise realizada a partir da distribuição dos bolsistas de produtividade em Psicologia do CNPq nos Estados nacionais ilustra que eles estão distribuídos em 19 estados do Brasil. A Região Sudeste é a que concentra o maior número de bolsistas - 161 (54,6\%), seguida pela Região Sul, com 53 pesquisadores bolsistas (18,6\%). Considerando-se por estado, predomina São Paulo, com 94 bolsistas (31,9\%), seguido por Rio de Janeiro $(15,6 \%)$, Rio Grande do Sul (14,2\%), Distrito Federal (10,2\%) e Minas Gerais (5,4\%). Os demais estados, somados, concentram $22,7 \%$ dos bolsistas.

Há uma discrepância no estado de São Paulo, quando comparado com os demais, no que se refere ao número de bolsistas de produtividade. Para tal, deve-se considerar que, das 53 IES com bolsistas de produtividade no Brasil, 13 (24,53\%) estão localizadas no Estado referido. Além de tal concentração, devem-se considerar as grandes desigualdades que o sistema educacional brasileiro apresenta entre as regiões do país. A oferta e a procura do ensino ocorrem de forma diferenciada em cada estado, em decorrência do nível socioeconômico desigual e das características peculiares de cada qual. São Paulo se destaca em relação aos demais devido à prioridade delegada ao setor educacional (Costa et al., 2012). O tempo médio de conclusão do ensino fundamental no estado, por exemplo, é constatado como o mais curto do país (9,1 anos). No que tange ao ensino superior, destaca-se que $54 \%$ dos alunos de graduação concentram-se na Região Sudeste, que abrange o Estado de São Paulo (Castro, 2000).

Tendo em vista as diferenças apresentadas em relação ao investimento em educação nos estados do Brasil, já era esperado que houvesse também uma desigualdade no que se refere à pós-graduação. Entre 1999 e 2006, a Região Sudeste contava com quase $60,0 \%$ dos bolsistas de produtividade em Psicologia (Hutz, Rocha, Spink, \& Menandro, 2012), o que pode justificar a concentração significativa de bolsistas no estado de São Paulo $(31,9 \%)$ (Costa et al., 2012). As verbas destinadas às Fundações de Amparo à Pesquisa dos estados também são bastante diversificadas. Em termos de comparação, a Fundação de Amparo à Pesquisa de São Paulo (FAPESP) desembolsou 2398 bilhões no triênio analisado, enquanto a Fundação de Amparo à Pesquisa do Rio Grande do Sul (FAPERGS) investiu 6,484 milhões no mesmo período, ou seja, um investimento aproximadamente 370 vezes menor (FAPERGS, 2011; Fapesp, 2012).

Apoiando os dados encontrados acerca da proeminência do Estado de São Paulo quanto ao amparo à pesquisa, a análise das cinco IES com o maior número de pesquisadores bolsistas de produtividade em Psicologia do CNPq resultou em um destaque da Universidade de São Paulo (USP), que comporta 50 pesquisadores (16,95\%). Esta é seguida pela Universidade de Brasília (UnB), com 27 bolsistas (9,15\%), Universidade Federal do Rio Grande do Sul (UFRGS), com 23 pesquisadores $(7,80 \%)$; Universidade Federal do Rio de Janeiro (UFRJ), com 15 (5,08\%); e Universidade do Estado do Rio de Janeiro (UERJ), com 12 bolsistas (4,07\%). Dessa forma, apenas cinco IES concentram 43,05\% do número total de bolsistas de produtividade em Psicologia do Brasil, destacando-se as Regiões Sudeste e Sul, o que corrobora os dados anteriormente discutidos.

Com referência às orientações realizadas pelos bolsistas de produtividade, percebe-se que a categoria 2F não apresenta nenhum orientando de pós-doutorado. Essa categoria de bolsa possui mé- 
dia de apenas 0,16 orientandos de doutorado e o menor número de mestrandos $(0,66)$. No entanto, possui o maior número de orientandos de iniciação científica $(7,5)$. Já os bolsistas da categoria 1 A apresentam maior número de orientandos de mestrado $(7,13)$, doutorado $(7,96)$ e pós-doutorado $(1,7)$. Os demais pesquisadores representam um número proporcionalmente maior de mestrandos e doutorandos do que de iniciação científica e pós-doutorandos.

É possível observar, a partir da Tabela 2, que a média de orientandos não se altera consideravelmente conforme a categoria de bolsa. Comparada à Tabela 3, que explana a média de publicações conforme a categoria de bolsa no triênio analisado, a média de publicações não está de acordo com a média de orientandos de cada bolsa. Por isso, não há uma aparente relação entre a produção e a formação de recursos humanos.

Uma das metas do novo Plano Nacional de PPG é aumentar o número de orientandos, o que poderia influenciar novos investimentos de programas, visando, idealmente, ao aumento de produção científica nacional (Capes, 2010). Contudo, o aumento de orientandos não necessariamente influenciará no aumento da produção científica.

A Tabela 3 ilustra a relação entre a categoria da bolsa CNPq (Sênior, 1A, 1B, 1C, 1D, 2 e 2F) e o Qualis (A1, A2, B1, B2. B3, B4, B5 e C) dos periódicos nos quais os artigos desses pesquisadores foram publicados no triênio 2009-2011. As colunas indicam o Qualis dos periódicos, enquanto as linhas representam as categorias das bolsas.

Os bolsistas de produtividade da categoria 1B, por exemplo, publicaram em média 11,98 artigos no triênio 2009-2011, sendo 1,38 artigos pertencentes à categoria Qualis A1. Ou seja, dentre a média de quase 12 artigos publicados ao longo dos três anos analisados, menos de dois artigos estão em revistas de Qualis A1.

Os pesquisadores da bolsa de categoria $1 \mathrm{~A}$ são os que mais publicaram no triênio analisado, contemplando uma média de 14,44 artigos no período. No entanto, essa é a categoria que apresentou maior índice de publicação em revistas de Qualis A1 $(1,83)$ e fora do sistema Qualis $(1,96)$.

Tabela 2

Média de orientações finalizadas e em andamento pelos pesquisadores bolsistas de produtividade em Psicologia do CNPq no triênio 2009 -2011

\begin{tabular}{|c|c|c|c|c|c|c|c|}
\hline Orientação & $S$ & $1 \mathrm{~A}$ & $1 \mathrm{~B}$ & $1 C$ & $1 \mathrm{D}$ & 2 & $2 \mathrm{~F}$ \\
\hline Iniciação científica & 4 & 4,36 & 4,03 & 5,00 & 5,28 & 4,21 & 7,50 \\
\hline Mestrado & 3 & 7,13 & 5,38 & 6,32 & 5,54 & 6,04 & 3,17 \\
\hline Doutorado & 3 & 7,96 & 5,79 & 5,28 & 5,28 & 3,94 & 0,66 \\
\hline Pós-doutorado & 1 & 1,70 & 0,86 & 0,72 & 0,36 & 0,16 & 0,00 \\
\hline
\end{tabular}

Nota: CNPq: Conselho Nacional de Desenvolvimento Científico e Tecnológico.

Tabela 3

Média trienal de artigos publicados pelos pesquisadores bolsistas de produtividade em Psicologia do CNPq no triênio $2009-2011$

\begin{tabular}{|c|c|c|c|c|c|c|c|c|c|c|c|}
\hline & & A1 & A2 & B1 & B2 & B3 & B4 & B5 & C & Sem Qualis & Média total \\
\hline \multirow{6}{*}{ 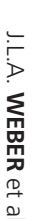 } & Sênior & 2,00 & 0,00 & 3,00 & 1,00 & 0,00 & 0,00 & 0,00 & 0,00 & 1,00 & 6,00 \\
\hline & $1 \mathrm{~A}$ & 1,83 & 2,78 & 2,39 & 2,35 & 1,13 & 1,26 & 0,52 & 0,22 & 1,96 & 14,44 \\
\hline & $1 \mathrm{~B}$ & 1,38 & 2,96 & 2,69 & 1,65 & 0,90 & 0,93 & 0,41 & 0,10 & 0,96 & 11,98 \\
\hline & $1 \mathrm{C}$ & 1,28 & 2,60 & 2,00 & 1,24 & 1,04 & 0,76 & 0,36 & 0,12 & 1,20 & 10,60 \\
\hline & $1 \mathrm{D}$ & 0,72 & 1,95 & 2,87 & 1,33 & 1,10 & 0,64 & 0,72 & 0,08 & 1,38 & 10,79 \\
\hline & 2 & 0,70 & 1,56 & 1,99 & 1,15 & 0,96 & 0,84 & 0,55 & 0,19 & 1,26 & 9,20 \\
\hline @ & $2 \mathrm{~F}$ & 0,33 & 1,00 & 1,33 & 0,33 & 1,33 & 0,83 & 0,17 & 0,00 & 0,33 & 5,65 \\
\hline & Média total & 1,18 & 1,84 & 2,32 & 1,29 & 0,92 & 0,75 & 0,39 & 0,10 & 1,16 & 9,81 \\
\hline
\end{tabular}

6 Nota: CNPq: Conselho Nacional de Desenvolvimento Científico e Tecnológico. 
A categoria 1B se aproxima da $1 \mathrm{~A}$ no índice de publicação Qualis A1, com uma média de 1,38 artigos publicados no triênio 2009-2011. Além disso, ultrapassa a categoria $1 \mathrm{~A}$ nas publicações com Qualis A2 (2,96, comparado à 2,78 na categoria $1 \mathrm{~A})$ e Qualis $B 1$ (2,69, comparado à média de 2,39 da categoria $1 \mathrm{~A}$ ).

Isso denota que, no triênio analisado neste estudo, os bolsistas de produtividade em Psicologia publicaram em grande quantidade. No entanto, a qualidade não está sendo priorizada, de acordo com os critérios da Capes que definem as diretrizes do sistema Qualis dos periódicos. Para Yamamoto et al. (2012), esse fenômeno denomina-se "produtivismo" na ciência, e ocorre principalmente devido à cultura acadêmica ainda predominante no Brasil, que leva muitos pesquisadores a priorizarem a quantidade de estudos em relação à sua qualidade ou relevância científica e social. Os órgãos que avaliam a pós-graduação, que deveriam fomentar esse debate, reforçam a busca por quantidade de publicações. Isso acaba por configurar um esvaziamento nas publicações, uma vez que a prioridade é "gerar números" (Waters, 2006).

A média total de artigos publicados em cada categoria do sistema Qualis, levando em consi- deração todos os bolsistas de produtividade - independentemente da categoria da bolsa -, é indicada na última linha da Tabela 3. Em média, os bolsistas publicaram 1,18 artigos em Qualis A1 no triênio analisado, e 2,32 no Qualis B1.

Em relação aos livros e capítulos de livros publicados pelos bolsistas, foi constatado que, diferentemente das publicações em periódicos, os pesquisadores com bolsa $1 \mathrm{~B}$ foram os que mais publicaram. Eles tiveram uma média trienal de 2,62 livros e 8,97 capítulos de livro publicados, enquanto os bolsistas 1A publicaram em média 2,52 livros e 7,04 capítulos. Inclusive, em relação ao número de capítulos de livros publicados, os pesquisadores 1D, com uma média de 7,97 capítulos, tiveram mais publicações do que os bolsistas $1 \mathrm{~A}$ e $1 \mathrm{C}$, que publicaram 7,04 e 4,36 capítulos respectivamente. Dessa forma, ainda que não haja um sistema tão claro de avaliação e classificação dessas obras, foi observada uma alta produção de livros e capítulos de livros.

Na Tabela 4 são apresentadas as análises de associação entre as variáveis tipo de bolsa do pesquisador e publicações nos diferentes Qualis, utilizando como categoria de referência os pesquisadores 2 e 2F. Em relação ao Qualis A1, todas as

\section{Tabela 4}

Análise de associação entre o tipo de bolsa e as publicações nos diferentes Qualis

\begin{tabular}{|c|c|c|c|c|c|c|}
\hline Qualis & OR/IC95\% & Sênior+1A & 1B & $1 C$ & 1D & $2+2 F(R E F)$ \\
\hline \multirow[t]{2}{*}{$A 1$} & $O R$ & $3,49^{*}$ & $2,35^{*}$ & $3,70^{*}$ & 0,90 & 1,00 \\
\hline & IC95\% & $1,38-8,85$ & $1,05-5,28$ & $1,47-9,30$ & $0,44-1,83$ & \\
\hline \multirow[t]{2}{*}{$\mathrm{A} 2$} & $O R$ & 1,68 & $2,69^{*}$ & 2,24 & 1,63 & 1,00 \\
\hline & IC95\% & $0,63-4,46$ & $0,98-7,41$ & $0,80-6,27$ & $0,74-3,56$ & \\
\hline \multirow[t]{2}{*}{ B1 } & $O R$ & 2,73 & 1,50 & 1,56 & 2,15 & 1,00 \\
\hline & IC95\% & $0,78-9,57$ & $0,58-3,89$ & $0,56-4,39$ & $0,84-5,44$ & \\
\hline \multirow[t]{2}{*}{ B2 } & $O R$ & $5,10^{*}$ & 1,62 & 0,79 & 1,46 & 1,00 \\
\hline & IC95\% & $1,47-17,71$ & $0,70-3,75$ & $0,34-1,82$ & $0,70-3,02$ & \\
\hline \multirow[t]{2}{*}{ B3 } & $O R$ & 1,35 & 0,93 & 0,90 & $2,04^{\star}$ & 1,00 \\
\hline & IC95\% & $0,57-3,18$ & $0,42-2,04$ & $0,39-2,09$ & $1,00-4,19$ & \\
\hline \multirow[t]{2}{*}{ B4 } & $O R$ & $2,62^{*}$ & 1,40 & 1,03 & 0,91 & 1,00 \\
\hline & IC95\% & $1,07-6,44$ & $0,64-3,08$ & $0,44-2,40$ & $0,45-1,84$ & \\
\hline \multirow[t]{2}{*}{ B5 } & $O R$ & 1,72 & 0,91 & 0,90 & 1,43 & 1,00 \\
\hline & IC95\% & $0,70-4,20$ & $0,37-2,28$ & $0,34-2,40$ & $0,68-3,02$ & \\
\hline \multirow[t]{2}{*}{ C } & $O R$ & 0,99 & 1,25 & 0,94 & 0,90 & 1,00 \\
\hline & IC95\% & $0,21-4,61$ & $0,34-4,63$ & $0,20-4,40$ & $0,25-3,30$ & \\
\hline
\end{tabular}

Nota: *Diferença significativa.

OR: Odds Ratio; IC95\%: Intervalo de Confiança de 95\%; REF: Referência. 
demais categorias (1A, 1B, 1C, 1D) de bolsas publicaram significativamente mais do que a bolsa de referência, com exceção da bolsa $1 \mathrm{D}$. A bolsa $1 \mathrm{~A}$ tem três vezes mais chance de publicar no Qualis A1 $(O R=3,49$, IC $95 \%=1,38-8,85)$ do que a categoria de referência (2 e 2F). Também existe diferença significativa nas categorias $1 \mathrm{~B}(O R=2,35, \mathrm{IC} 95 \%=$ $1,05-5,28)$ e $1 \mathrm{C}(O R=3,70$, IC95\% = 1,47-9,30) em relação ao Qualis A1. Esses dados reforçam a ideia de que a categoria 1D, de fato, é a categoria de "entrada" para outra classe de bolsistas no que diz respeito à produção.

Quanto às demais categorias de Qualis, foi observado que a bolsa $1 \mathrm{~A}$ publica significativamente mais no Qualis B2, com a maior diferença entre todas as categorias analisadas, possuindo cinco vezes mais chances de publicar ( $O R=5,10, I C 95 \%$ $=1,47-17,71)$. Foi constatado também que a bolsa 1A publica significativamente mais no Qualis B4 $(O R=2,62, I C 95 \%=1,07-6,44)$. Na categoria Qualis A2, a bolsa 1B foi a única a apresentar uma diferença significativa $(O R=2,69$, IC95\% $=0,98-7,41)$. Já a bolsa 1D apresentou diferença significativa apenas na categoria $B 3$, na qual possui duas vezes mais chance de publicação $(O R=2,04$, IC95\% = 1,00 - 4,19). Por meio da presente análise estatística, não foram encontradas diferenças significativas nas publicações de Qualis B1, B5 e C entre as categorias de bolsistas.

No entanto, os resultados acima referidos - a média numérica e a análise estatística -, precisam ser ponderados. O produtivismo e os critérios não padronizados de avaliação editorial podem gerar vieses. Por isso, pode ser interessante considerar outros critérios de avaliação da produção de cada pesquisador, como, por exemplo, a análise do índice $\mathrm{h}$ das publicações.

O índice h, proposto por Hirsch em 2005, é um instrumento que se propõe a medir o impacto cumulativo de um autor no meio acadêmico, verificando o número de citações que suas publicações receberam. Por meio dessa análise, é gerado um número métrico de impacto acadêmico, o qual combina quantidade com qualidade das publicações. Um pesquisador não pode ter um alto índice $h$ sem

8 publicar um grande número de artigos, porém tais artigos devem ser muito citados para impactarem positivamente no índice h. O programa Publish or Perish usa a base de dados do Google Acadêmico para obter as citações brutas referentes ao histórico de produção do autor, e então as analisa e fornece estatísticas relacionadas (Harzing, 2008). Para o presente estudo, foram utilizados os dados referentes à média de artigos e citações, assim como o índice h de cada pesquisador.

$\mathrm{O}$ índice $\mathrm{h}$ dos pesquisadores bolsistas foi encontrado por meio do nome de citação que eles utilizam na Plataforma Lattes. Caso o pesquisador tivesse mais de um nome de citação, foram considerados os índices referentes ao nome mais utilizado nos últimos artigos publicados. Além disso, não foram avaliados os pesquisadores que, em decorrência de um nome de citação muito comum, ou com muitos homônimos, obtiveram resultados superiores a 1 mil artigos publicados. Assim, foram excluídos desta etapa da análise 37 pesquisadores.

Os dados da Tabela 5 apresentam a média de artigos publicados, a média de citações globais e o índice $h$ médio por categoria ao longo da carreira acadêmica dos pesquisadores. A partir dessa tabela, pôde-se constatar que tanto a média de artigos publicados quanto a média de citações globais e o índice h médio aumentam conforme a categoria e o nível da bolsa de produtividade, sendo a categoria $2 \mathrm{~F}$ a que apresentou a menor média nos três índices $(27,8 ; 150,8$ e 5,4, respectivamente), enquanto a categoria $1 \mathrm{~A}$ foi a que obteve a maior média (148; 804,52 e 12,78 , respectivamente).

A única representante na categoria Sênior apresentou o maior índice de citações (1 152) e o maior índice $h$ (19). Esse dado demonstra que, mesmo com número de publicações um pouco abaixo da categoria 1A (148), a bolsista da categoria Sênior (com 121 publicações) tem um índice h consideravelmente mais elevado do que a média dos bolsistas $1 \mathrm{~A}(12,78)$. Esse dado pode denotar uma qualidade maior nas publicações dessa bolsista (avaliada pela ferramenta Qualis) e/ou maior número de citações, que acarreta o aumento do seu índice h.

Quanto ao que se refere às áreas de interesse destacadas nos currículos Lattes dos pesquisadores da amostra analisada, foi constatado que a Psico- 
Tabela 5

Médias do fator de impacto, publicações e citações dos pesquisadores bolsistas de produtividade em Psicologia do CNPq ao longo de sua carreira acadêmica

\begin{tabular}{lccc}
\hline Categoria da bolsa & Média de artigos & Média de citações & Fator de impacto (h) \\
\hline 2F & 27,80 & 150,80 & 5,40 \\
2 & 74,35 & 332,38 & 8,63 \\
1D & 75,73 & 535,67 & 9,24 \\
1C & 89,71 & 579,86 & 12,09 \\
1B & 101,88 & 632,46 & 12,77 \\
1A & 148,00 & 804,52 & 12,78 \\
\hline Sênior & 121,00 & 1152,00 & 19,00 \\
\hline Total & 84,39 & 450,51 & 9,76 \\
\hline
\end{tabular}

Nota: CNPq: Conselho Nacional de Desenvolvimento Científico e Tecnológico.

logia do Desenvolvimento foi a mais citada (79 vezes), seguida pela Psicologia Social (74), Tratamento e Prevenção Psicológica (68), Fundamentos e Medidas de Psicologia (57) e Psicologia Experimental (48). Considerando que foram analisados os currículos de 295 pesquisadores, cada um deles cita em média 2,34 áreas de interesse, o que resulta em um total de 690 citações. Essas cinco áreas de interesse citadas representam $47,2 \%$ do total, sendo que os $52,8 \%$ restantes se dividem em 140 áreas diferentes.

Verifica-se uma diferença razoável entre a primeira área (Psicologia do Desenvolvimento) mais citada e a quinta (Psicologia Experimental), havendo uma discrepância de 31 citações entre uma e outra. Esse número pode ser considerado alto, tendo em vista que o total de pesquisadores é de 295.

Dessa forma, no que diz respeito às áreas de produção dos pesquisadores, observa-se a diversidade própria da Psicologia. Esta apresenta como característica constitutiva o somatório de diversas práticas e saberes que comportam diferentes definições de produção do conhecimento. Assim, os objetos de estudo da Psicologia, bem como seus métodos de pesquisa, são diversificados. Por esse motivo, há uma considerável heterogeneidade de subáreas (Lo Bianco et al., 2010).

Este estudo ainda considerou em sua análise 2620 publicações em 724 periódicos, tanto nacionais (322 publicações) quanto internacionais (84). Os sete principais periódicos com maior número de publicações dos pesquisadores bolsistas de produ- tividade em Psicologia do CNPq no triênio analisado foram: de origem nacional - "Psicologia: Reflexão e Crítica" (106 publicações), "Psicologia: Teoria e Pesquisa" (102), "Psicologia: Ciência e Profissão" (57) e "Psico" (PUCRS) (57); e de origem internacional - "Interamerican Journal of Psychology" (42), "Acta Comportamentalia" (22) e "The Spanish Journal of Psychology" (20).

A partir desses dados, evidencia-se uma diferença importante entre o número de publicações nos principais periódicos de origem nacional e internacional, que concentram $12,29 \%$ e $3,21 \%$ das publicações, respectivamente. Também é destacado o fato de os dois periódicos nacionais com o maior número de artigos publicados apresentarem um número significativamente maior do que os restantes.

Os dados indicam uma produção predominantemente nacional e difusa, uma vez que as publicações em Psicologia são distribuídas em 724 periódicos diferentes, havendo uma média geral de 3,62 artigos publicados em cada um. É importante ressaltar que a Psicologia é uma área que está interligada a diversos campos de conhecimento, o que explica o elevado número de artigos publicados em periódicos de outras áreas.

A partir da análise dos dados encontrados, pôde-se perceber que os bolsistas de produtividade científica em Psicologia do CNPq possuem características diversificadas, não permitindo que seja traçado um perfil único dos mesmos. Como era de se esperar, predominam os pesquisadores da cate- 
goria 2, distribuídos, em sua maioria, entre as áreas da Psicologia do Desenvolvimento e da Psicologia Social. Além disso, a maior parte dos pesquisadores é do sexo feminino e encontra-se, sobretudo, na Região Sudeste do país, em especial no estado de São Paulo, que concentra uma parcela de bolsistas significativamente maior que os demais estados. Esse resultado é semelhante ao encontrado no estudo de Wendt, DeSousa, Lisboa e Koller (2013), que também busca traçar o perfil dos bolsistas de produtividade em Psicologia, utilizando como base os anos entre 2007 e 2011 para análise dos dados dos Currículos Lattes dos pesquisadores.

Este estudo teve como base o triênio 2009-2011 e complementa a análise dos dados encontrados em Wendt et al. (2013), ampliando o conhecimento acerca das contribuições dos pesquisadores para a produção de conhecimento em Psicologia, e considerando as limitações indicadas por aqueles autores, tais como a avaliação das áreas temáticas das publicações dos bolsistas de produtividade, a avaliação do extrato Qualis dos periódicos onde publicaram, a proporção de estudos internacionais e o tempo de doutoramento. Além dessas contribuições, o presente estudo também tenta refletir sobre elementos de qualidade da produção acadêmica desses pesquisadores, utilizando elementos da relação entre quantidade e qualidade da produção.

Um dos quesitos principais para avaliação dos PPG em Psicologia é a quantidade de produções, mais evidentemente do que a qualidade destas. Considerando o presente fato, fez-se necessário refletir sobre tal produtivismo, já que os parâmetros atualmente utilizados supervalorizam a quantidade em detrimento da qualidade ou do benefício acadêmico e/ou social. Entretanto, as médias encontradas para o índice $\mathrm{h}$ puderam proporcionar uma reflexão acerca da qualidade das publicações, visto que, quando comparadas às categorias e aos níveis de bolsa, observou-se que algumas categorias, apesar de terem um número maior de publicações, apresentaram índice h praticamente igual a categorias que publicaram menos. Isso demonstra que o índice 10 h leva em conta não somente a quantidade de arti- gos publicados, mas também seu impacto social e acadêmico. Percebe-se que há uma coerência nos critérios de avaliação da Capes para o fornecimento de bolsas, pois as que possuem nível mais elevado publicam significativamente mais nos periódicos com melhor avaliação Qualis. Além disso, o índice h cresce de acordo com o nível da bolsa, das categorias de 2 a 1A, de modo que a existência de um único caso - a pesquisadora na categoria Sênior, com o maior índice h da área -, retrata situação muito particular para suscitar maiores generalizações a respeito da categoria específica.

Vale ressaltar que esta pesquisa apresenta algumas limitações, como a análise dos pesquisadores bolsistas por meio apenas dos currículos Lattes (o que restringiu as informações às atualizações feitas por eles) e a ocorrência de homônimos, que acarretou a exclusão de 37 pesquisadores na análise do índice h. Apesar dessas limitações, o presente estudo incitou reflexões que foram além do perfil dos bolsistas de produtividade em Psicologia, questionando a maneira pela qual os PPG em Psicologia são avaliados bem como as políticas de produção científica no país. É importante que se realizem mais estudos nessa área, visando a um investimento global nas políticas de ensino superior, a uma mudança na condução dos PPG e à melhoria de qualidade das publicações científicas.

\section{Referências}

Castro, M. H. G. (2000). As desigualdades regionais no sistema educacional brasileiro. In R. Henriques. Desigualdade e pobreza no Brasil. Rio de Janeiro: IPEA.

Conselho Federal de Psicologia. (2012). Cadastro Nacional de Psicólogos. Recuperado em outubro 14, 2012, de http://cadastro.cfp.org.br/siscafweb/carrega Conselho. do? tipoAcesso $=4 \& s=1 \&$ tipoConsulta=pf\&controle $=$ $0 \&$ sigla $=$ cfp\&ini $=1$

Conselho Nacional de Desenvolvimento Científico e Tecnológico. (2006a). Institucional: o CNPq. Recuperado em outubro 14, 2012, de http://www.cnpq.br/ web/guest/o-cnpq

Conselho Nacional de Desenvolvimento Científico e Tecnológico. (2006b). Mapa de investimentos do CNPq. Recuperado em outubro 14, 2012, de http:// efomento.cnpq.br/efomento/distribuicaoGeografica/ distribuicaoGeografica.do?metodo=apresentar 
Conselho Nacional de Desenvolvimento Científico e Tecnológico. (2006c). Bolsas no País: produtividade em pesquisa - PQ. Recuperado em outubro 23, 2013, de http://www.cnpq.br/web/guest/apresentacao13

Coordenação de Aperfeiçoamento de Pessoal de Nível Superior. (2006). História e missão. Recuperado em outubro 14, 2012, de http://capes.gov.br/sobre-acapes/historia-e-missao

Coordenação de Aperfeiçoamento de Pessoal de Nível Superior. (2010). Plano Nacional de Pós-Graduação 2011-2020. Brasília: Capes.

Costa, J. P., Amorim, K. M. O., Pessanha, V. C., \& Yamamoto, O. H. (2012). Quem estuda a profissão de psicólogo no Brasil? Arquivos Brasileiros de Psicologia, 24(2), 2-18. Recuperado em março 21, 2013, de http:// pepsic.bvsalud.org/ pdf/arbp/v64n2/v64n2a02.pdf

Fundação de Amparo à Pesquisa do Estado do Rio Grande do Sul. (2011). Relatório exercício 2011. Recuperado em novembro 17, 2012, de http://www.fapergs. rs.gov.br/upload/20120621104014relato_fapergs_ 2011.pdf

Fundação de Amparo à Pesquisa do Estado de São Paulo (27 mar. 2012). Diário oficial do Estado de São Paulo: relatório da administração. São Paulo: Imprensa Oficial. Seção 1, 122(58), 41. Recuperado em novembro 17, 2012, de http://www.fapesp.br/estatisticas/ balancos/Balanco_de_2011-1-pg_0041_do_DOE_de_ 27032012.pdf

Harzing, A. W. (2008). Publish or Perish: Reflections on the $h$-index. Retrieved on December 11, 2012, from http://www. harzing.com/pop_hindex.htm

Hutz, C. S., Rocha, M. L., Spink, M. J. P., \& Menandro, P. R. M. (2010). Perfil, avaliação e metas de produção intelectual dos Programas de Pós-Graduação em Psicologia. Psicologia: Reflexão Crítica, 23(Supl 1), 25-34.
http://dx.doi.org/10.1590/S0102-797220100004 00004

Leta, J. (2003). As mulheres na ciência brasileira: crescimento, contrastes e um perfil de sucesso. Estudos Avançados, 17(49), 271-284. http://dx.doi.org/ 10.1590/S0103-40 142003000300016

Lo Bianco, A. C., Almeida, S. S., Koller, S. H., \& Paiva, V. (2010). A internacionalização dos programas de pós-graduação em Psicologia: perfil e metas de qualifıcação. Psicologia: Reflexão e Crítica, 23(Supl 1), 1-10. http://dx.doi.org/10.1590/S0102-79722010000 400002

Mendes, P. H. C., Martelli, D. R. B., Souza, W. P., Quirino Filho, S., \& Martelli Júnior, H. (2010). Perfil dos pesquisadores bolsistas de produtividade científica em medicina no CNPq, Brasil. Revista Brasileira de Educação Médica, 34(4), 535-541. http://dx.doi.org/ 10.1590/S0100-55022010000400008

Thomaz, P G., Assad, R. S., \& Moreira, L. F. P (2011). Uso do fator de impacto e do índice $\mathrm{H}$ para avaliar pesquisadores e publicações. Arquivos Brasileiros de Cardiologia, 96(2), 90-93. doi: http://dx.doi.org/10.1590/s0 066-782X2011000 200001

Waters, L. (2006). Inimigos da esperança: publicar, parecer e o eclipse da erudição. São Paulo: Unesp.

Wendt, G. W., Lisboa, C. S. M., DeSousa, D. A., \& Koller, S. H. (2013). Perfil dos Bolsistas de Produtividade em Pesquisa do CNPQ em Psicologia. Psicologia: Ciência e Profissão, 33(3), 536-547. http://dx.doi.org/10.1590/ S1414-98932013000300003

Yamamoto, O., Tourinho, E., Bastos, A. V. B., \& Menandro, P. (2012). Produção científica e "produtivismo": há alguma luz no final do túnel? Revista Brasileira de Pós-Graduação, 9(18), 727-750.

Recebido: dezembro 20, 2012

Versão final: dezembro 16, 2013

Aprovado: março 6, 2014 
